\title{
Tumor size interpretation for predicting cervical lymph node metastasis using a differentiated thyroid cancer risk model
}

\author{
Rong-liang Shi ${ }^{1-3, *}$ \\ Ning $\mathbf{Q u}^{1,2, *}$ \\ Shu-wen Yang ${ }^{1,2}$ \\ Ben $\mathrm{Ma}^{1,2}$ \\ Zhong-wu Lu' ${ }^{1,2}$ \\ Duo Wen ${ }^{1,2}$ \\ Guo-hua Sun ${ }^{1,2}$ \\ Yu Wang ${ }^{1,2}$ \\ Qing-hai ji ${ }^{1,2}$

\begin{abstract}
'Department of Head and Neck Surgery, Fudan University Shanghai Cancer Center, ${ }^{2}$ Department of Oncology, Shanghai Medical College, Fudan University, ${ }^{3}$ Department of General Surgery, Minhang Hospital, Fudan University, Shanghai, People's Republic of China

*These authors contributed equally to this work
\end{abstract}

This article was published in the following Dove Press journal:

OncoTargets and Therapy

12 August 2016

Number of times this article has been viewed

\begin{abstract}
Lymph node metastasis (LNM) is common in differentiated thyroid cancer (DTC), but management of clinically negative DTC is controversial. This study evaluated primary tumor size as a predictor of LNM. Multivariate logistic regression analysis was used for DTC patients who were treated with surgery between 2002 and 2012 in the Surveillance, Epidemiology, and End Results (SEER) database, to determine the association of tumor size at $10 \mathrm{~mm}$ increments with LNM. A predictive model was then developed to estimate the risk of LNM in DTC, using tumor size and other clinicopathological characteristics identified from the multivariate analysis. We identified 80,565 eligible patients with DTC in the SEER database. Final histology confirmed 9,896 (12.3\%) cases affected with N1a disease and 8,194 (10.2\%) cases with N1b disease. After the patients were classified into subgroups by tumor size, we found that the percentages of male sex, white race, follicular histology, gross extrathyroidal extension, lateral lymph node metastasis, and distant metastasis gradually increased with size. In multivariate analysis, tumor size was a significant independent prognostic factor for LNM; in particular, the odds ratio for lateral lymph node metastasis continued to increase by size relative to a $1-10 \mathrm{~mm}$ baseline. The coefficient for tumor size in the LNM predictive model was $\sim 0.20$, indicating extra change in $\log$ (odds ratio) for LNM as 0.2 per unit increment in size relative to baseline. In conclusion, larger tumors are likely to have aggressive features and metastasize to a cervical compartment. Multistratification by size could provide more precise estimates of the likelihood of LNM before surgery.
\end{abstract}

Keywords: differentiated thyroid cancer, size, lymph node metastasis, risk, SEER

\section{Introduction}

In many regions of the world, the incidence of thyroid cancer (TC) is increasing faster than that of any other malignancy. ${ }^{1,2}$ Differentiated thyroid cancer (DTC) corresponds to $>90 \%$ of TCs. This type of TC tends to have an indolent clinical course with low morbidity and mortality. ${ }^{3}$ Although DTC is associated with a relatively good prognosis, ${ }^{4} \sim 10 \%$ of patients with DTC die of this disease. Therefore, DTC has a wide spectrum of biologic and clinical behaviors that can result in tumor recurrence and death; these mainly depend on the patient's characteristics and tumor features at the time of diagnosis. In as many as $53 \%$ of patients with DTC, cervical lymph node metastasis (LNM) is found at the time of initial surgery. ${ }^{1-5}$ Several studies have reported increased mortality rates among patients with LNM; other studies have reported shorter survival. ${ }^{6-8}$ Thus, precise prediction of LNM at the time of initial treatment would help to determine the optimal treatment plan and aid in the decision-making process regarding the extent of surgery needed. Preoperative imaging methods, including 
neck ultrasonography and computed tomography, could be applied for comprehensive and objective evaluation of the neck to evaluate the status of lymph nodes preoperatively. ${ }^{7,9}$ Primary tumor size is the main factor in evaluating DTC because large tumors are significantly more aggressive than the small ones. ${ }^{10,11}$ According to the 2010 tumor-nodemetastasis (TNM) classification of the American Joint Committee on Cancer/International Union Against Cancer, tumor size, extrathyroid extension (ETE), and LNM are the three important factors for evaluating the clinical outcomes of DTC patients. ${ }^{12,13}$ Interestingly, it is thought that the effects of ETE and LNM vary by tumor size. ${ }^{11,14}$

In this study, we divided DTC patients into six groups based on tumor size, with the objective of evaluating the effect of tumor size on the probability of developing LNM. We then developed a risk calculator based on tumor size and other risk factors to predict LNM risk using data recorded between 2002 and 2012 in the Surveillance, Epidemiology, and End Results (SEER) Program. Ultimately, our goal was to develop a model that could allow us to make more informed decisions regarding the cervical management of DTC patients pre- and intraoperatively when initiating treatment.

\section{Materials and methods}

\section{Patient selection from the SEER database}

We extracted data from the SEER cancer registry to conduct this study. The SEER, a population-based registry sponsored by the National Cancer Institute, collects information on cancer incidence and survival from 17 population-based cancer registries, including $\sim 28 \%$ of the population in the US. ${ }^{15}$ The data obtained from the SEER database contain no identifiers and are publicly available for studies on cancerbased epidemiology and health policy. The National Cancer Institute's SEER*Stat software (version 8.1.2; Surveillance Research Program, National Cancer Institute SEER*Stat software, www.seer.cancer.gov/seerstat) was used to identify patients with single primary DTC who were diagnosed between 2002 and 2012. We included patients diagnosed with DTC only, or with DTC as the first among other malignancies, who underwent surgery. Histological types were limited to papillary cancer $(n=52,891)$, follicular variant of papillary TC $(n=22,900)$, and follicular cancer $(n=4,774)$. We excluded patients with insufficient data or unknown clinicopathological profiles, undetermined histologies, or other types of TC (eg, medullary TC, anaplastic TC).

\section{Ethics statement}

An independent ethics committee/institutional review board at Fudan University Shanghai Cancer Center approved our study. This study was conducted in accordance with the Declaration of Helsinki and the reporting guidelines for this type of study. Informed patient consent is not necessary when using data released from the SEER database because these data are de-identified. We received permission to access the research data file in the SEER program from the National Cancer Institute, USA (reference number 13579-Nov2014).

\section{Assessment of clinicopathological variables and statistical analysis}

The regional lymph node metastases were classified into N1a (level VI [pretracheal, paratracheal, and prelaryngeal/Delphian lymph node metastases]) and N1b (unilateral, bilateral, or contralateral levels I, II, III, IV, or V or retropharyngeal or superior mediastinal lymph node (level VII) metastases). The following variables were used to analyze the risk factors for LNM: sex, age at diagnosis, maximal tumor size, and race (non-Hispanic white, and others [American Indian/Alaska Native, Asian/ Pacific Islander]), as provided by the SEER database. ${ }^{13}$ Continuous variables, such as age and maximal tumor size, were first compared and then converted to dichotomous variables with a cutoff point for further investigation in case the initial comparison indicated a significant difference. There were relatively few patients with larger tumors ( $\geq 51 \mathrm{~mm})$, and thus, these were grouped together $(n=4,774 ; 5.9 \%)$; the other patients were stratified into groups of $10 \mathrm{~mm}$ size as follows: $1-10 \mathrm{~mm}$, $11-20 \mathrm{~mm}, 21-30 \mathrm{~mm}, 31-40 \mathrm{~mm}, 41-50 \mathrm{~mm}$, and $\geq 50 \mathrm{~mm}$. According to the current staging system, the age of 45 years was used as the cutoff point to categorize patients as younger (age $<45$ years) or older (age $>45$ years). ${ }^{12}$ Multifocal primary lesions were defined as two or more cancer sites within the thyroid gland; a solitary intrathyroidal primary lesion represented only a single cancer site within the thyroid gland. An ETE of primary tumor is defined as disease that extends outside of the thyroid gland. It is classified as three types, thyroid capsule extension, minimal ETE (eg, tumor extending into strap muscle), and gross ETE (eg, tumor extending into esophagus, larynx, sternocleidomas muscle). The existence of multifocal lesions and ETE were both established on the final pathology assessment. The endpoint of the present study was DTC causespecific survival (CSS), which was calculated from the day of diagnosis to the day of cancer-specific death and was shown as "SEER cause-specific survival" in the SEER database. We followed the guidance of the 2010 TNM classification of the American Joint Committee on Cancer. ${ }^{12,13}$

Clinicopathological variables were described using counts, and categorical variables were described using percentages. Statistical analyses were performed using the chi-square $\left(\chi^{2}\right)$ test as appropriate. The odds ratios (ORs) for 
relationships between clinicopathological factors and LNM were calculated using binary logistic regression. A final model selection was performed by a backward stepdown selection process with the Akaike information criterion. ${ }^{16}$ The discrimination and calibration of the system were then assessed and internally validated using bootstrapping techniques. Two-sided $P$-values $<0.05$ were considered significant. All statistical analyses were performed using the Regression Modeling Strategy package ${ }^{17}$ in $\mathrm{R}$ version 3.2.2 (http://www.r-project.org/).

\section{Results}

\section{Baseline characteristics and comparison among groups by tumor size}

We identified 80,565 eligible patients with DTC in the SEER database between 2002 and 2012. The smallest group was that of patients with large tumors ( $\geq 51 \mathrm{~mm}$; $n=4,467)$. Most patients were classified into $1-10 \mathrm{~mm}(\mathrm{n}=31,721), 11-20 \mathrm{~mm}$ $(\mathrm{n}=22,786), 21-30 \mathrm{~mm}(\mathrm{n}=12,077), 31-40 \mathrm{~mm}(\mathrm{n}=6,031)$, and 41-50 mm $(\mathrm{n}=2,254)$ tumor size groups (Table 1$)$. The proportions of older patients and multifocality in each group were comparable with slight variations; however, the proportions of male sex, white race, follicular histology, gross ETE, lateral lymph node metastasis (LLNM), and distant metastasis gradually increased with increasing tumor size (Table 1). Although the proportion of patients who received total thyroidectomy/near-total thyroidectomy or radioisotopes varied slightly, the ratio of cancer-specific deaths increased significantly among the different groups (Table 1).

\section{Risk factors for cervical LNM}

According to the final pathology, 9,896 (12.3\%) patients had LNM involving only the central compartment (N1a) and $8,194(10.2 \%)$ had involvement of both the central and lateral compartments (N1b). Univariate and multivariate logistic regression analyses showed that LNM risk was significantly increased with male sex, older age ( $\geq 45$ years), larger tumor size, non-Hispanic white race, papillary histology type,

Table I Clinicopathological characteristics of DTC patients according to tumor size in the SEER database

\begin{tabular}{|c|c|c|c|c|c|c|c|c|c|c|c|c|c|c|c|}
\hline \multirow{3}{*}{$\begin{array}{l}\text { Characteristic } \\
\text { Total }\end{array}$} & \multirow{2}{*}{\multicolumn{2}{|c|}{$\begin{array}{l}\text { All patients } \\
\mathbf{N}=\mathbf{8 0 , 5 6 5}\end{array}$}} & \multicolumn{12}{|c|}{ Tumor size (mm) } & \multirow[t]{3}{*}{$P$-value } \\
\hline & & & \multicolumn{2}{|l|}{$1-10$} & \multicolumn{2}{|l|}{$I I-20$} & \multicolumn{2}{|l|}{$21-30$} & \multicolumn{2}{|l|}{$31-40$} & \multicolumn{2}{|l|}{$4 I-50$} & \multicolumn{2}{|l|}{$\geq \mathbf{5}$ I } & \\
\hline & Number & $\%$ & Number & $\%$ & Number & $\%$ & Number & $\%$ & Number & $\%$ & Number & $\%$ & Number & $\%$ & \\
\hline & 80,565 & 100 & 31,721 & 39.4 & 22,786 & 28.3 & 12,077 & 15.0 & 6,031 & 7.5 & 3,483 & 4.3 & 4,467 & 5.5 & \\
\hline \multicolumn{16}{|l|}{ Sex } \\
\hline Female & 62,219 & 77.2 & 25,842 & 81.5 & 18,033 & 79.1 & 9,096 & 75.3 & 4,247 & 70.4 & 2,254 & 64.7 & 2,747 & 61.5 & 0.001 \\
\hline Male & 18,346 & 22.8 & 5,879 & 18.5 & 4,753 & 20.9 & 2,981 & 24.7 & $\mathrm{I}, 784$ & 29.6 & 1,229 & 35.3 & $\mathrm{I}, 720$ & 38.5 & \\
\hline \multicolumn{16}{|l|}{ Age } \\
\hline$\geq 45$ years & 49,101 & 60.9 & 21,227 & 66.0 & 13,180 & 57.8 & 6,563 & 54.3 & 3,331 & 55.2 & 1,938 & 55.6 & 2,862 & 64.1 & 0.001 \\
\hline$<45$ years & 31,464 & 39.1 & 10,494 & 34.0 & 9,606 & 42.2 & $5,5 \mid 4$ & 45.7 & 2,700 & 44.8 & $\mathrm{I}, 545$ & 44.4 & 1,605 & 35.9 & \\
\hline \multicolumn{16}{|l|}{ Race } \\
\hline Blacks & 5,161 & 6.4 & 1,983 & 6.3 & $\mathrm{I}, \mathrm{I} 28$ & 5.0 & 722 & 6.0 & 483 & 8.0 & 324 & 9.3 & 521 & II.7 & $0.00 \mathrm{I}$ \\
\hline Whites & 66,042 & 82.0 & 26,517 & 83.6 & 18,789 & 82.5 & 9,793 & 81.1 & 4,802 & 79.6 & 2,705 & 77.7 & 3,436 & 76.9 & \\
\hline Other $^{\mathrm{a}}$ & 8,439 & 10.5 & 2,838 & 8.9 & 2,622 & 11.5 & 1,416 & 11.7 & 677 & 11.2 & 421 & 12.1 & 465 & 10.4 & \\
\hline \multicolumn{16}{|l|}{ Histology type } \\
\hline Papillary & 52,891 & 65.7 & 22,790 & 71.8 & $|5,37|$ & 67.5 & 7,443 & 61.6 & 3,197 & 53.0 & $\mathrm{I}, 809$ & 51.9 & $2,28 I$ & 51.1 & 0.001 \\
\hline FV-PTC & 22,900 & 28.4 & 8,605 & 27.1 & 6,559 & 28.8 & 3,492 & 28.9 & 1,906 & 31.6 & 1,033 & 29.7 & $\mathrm{I}, 305$ & 29.2 & \\
\hline Follicular & 4,774 & 5.9 & 326 & 1.0 & 856 & 3.8 & 1,142 & 9.5 & 928 & 15.4 & 641 & 18.4 & 881 & 19.7 & \\
\hline \multicolumn{16}{|l|}{ Extension } \\
\hline Capsule & 6,166 & 7.7 & $|, 06|$ & 3.3 & $\mathrm{I}, 753$ & 7.7 & 1,389 & 11.5 & 804 & 13.3 & 558 & 16.0 & 601 & 13.5 & 0.001 \\
\hline Minimal & 10,016 & 12.4 & 1,658 & 5.2 & 3,679 & 16.1 & 2,155 & 17.8 & 1,028 & 17.0 & 715 & 20.5 & 781 & 17.5 & \\
\hline Gross & 2,060 & 2.6 & 165 & 0.5 & 528 & 2.3 & 486 & 4.0 & 291 & 4.8 & 228 & 6.5 & 362 & 8.1 & \\
\hline Multifocality & 31,752 & 39.4 & 10,935 & 34.5 & 10,359 & 45.5 & 5,130 & 52.5 & 2,384 & 39.5 & $|, 33|$ & 38.2 & 1,613 & 36.1 & 0.001 \\
\hline \multicolumn{16}{|l|}{$\mathrm{N}$ stage } \\
\hline Total & 18,090 & 22.5 & 3,871 & 12.2 & 6,185 & 27.1 & 3,798 & 31.4 & 1,820 & 30.2 & $\mathrm{I}, 127$ & 32.4 & 1,289 & 28.9 & 0.001 \\
\hline $\mathrm{Nla}$ & 9,896 & 12.3 & 2,168 & 6.8 & 3,606 & 15.8 & 2,116 & 17.5 & 982 & 16.3 & 528 & 15.2 & 496 & II.I & 0.001 \\
\hline NIb & 8,194 & 10.2 & $\mathrm{I}, 703$ & 5.4 & 2,579 & 11.3 & 1,682 & 13.9 & 838 & 13.9 & 599 & 17.2 & 793 & 17.8 & 0.001 \\
\hline Distant metastasis ${ }^{\mathrm{b}}$ & 956 & 1.2 & 102 & 0.3 & 169 & 0.7 & 183 & 1.5 & 129 & 2.1 & 103 & 3.0 & 270 & 6.0 & 0.001 \\
\hline TT or near-TT & 67,391 & 83.6 & 24,058 & 75.8 & 20,654 & 90.6 & 10,778 & 89.2 & 5,276 & 87.5 & 3,070 & 88.1 & 3,555 & 79.6 & NS \\
\hline Radioisotopes & 38,912 & 48.3 & 9,067 & 28.6 & 13,679 & 60.0 & 7,803 & 64.6 & 3,869 & 64.2 & 2,230 & 64.0 & 2,264 & 50.7 & NS \\
\hline Cancer death & 630 & 0.8 & 67 & 0.2 & 83 & 0.4 & 124 & 1.0 & 91 & 1.5 & 67 & 1.9 & 198 & 4.4 & 0.001 \\
\hline
\end{tabular}

Notes: Data are presented as n (\%). ${ }^{a}$ Including American Indian/Alaska Native, Asian/Pacific Islander; bincluding the lungs, bones, and brain.

Abbreviations: DTC, differentiated thyroid cancer; FV-PTC, follicular variant of papillary thyroid cancer; NS, none significant; SEER, Surveillance Epidemiology and End Results; TT, total thyroidectomy. 
Table 2 Univariate and multivariate logistic regression analyses for cervical LNM in DTC from the SEER database

\begin{tabular}{|c|c|c|c|c|}
\hline \multirow[t]{2}{*}{ Independent variable } & \multicolumn{2}{|l|}{ Univariate } & \multicolumn{2}{|l|}{ Multivariate } \\
\hline & OR $(95 \% \mathrm{CI})$ & $P$-value & OR (95\% Cl) & $P$-value \\
\hline Sex (female vs male) & $0.579(0.558-0.60 \mathrm{I})$ & 0.001 & $0.601(0.576-0.627)$ & 0.001 \\
\hline Age $(\geq 45$ vs $<45)$ & $0.555(0.537-0.574)$ & 0.001 & $0.506(0.487-0.526)$ & 0.001 \\
\hline \multicolumn{5}{|l|}{ Tumor size $(\mathrm{mm})$} \\
\hline $1-10$ & I (Reference) & & I (Reference) & \\
\hline $1 \mathrm{I}-20$ & $2.680(2.564-2.803)$ & 0.001 & $2.101(2.033-2.204)$ & 0.001 \\
\hline $21-30$ & $3.300(3.136-3.473)$ & 0.001 & 2.601 (2.458-2.752) & 0.001 \\
\hline $31-40$ & $3.109(2.915-3.316)$ & 0.001 & $2.669(2.48 I-2.87 I)$ & 0.001 \\
\hline $4 I-50$ & $3.442(3.182-3.723)$ & 0.001 & $2.811(2.567-3.078)$ & 0.001 \\
\hline$\geq 50$ & $2.918(2.713-3.139)$ & 0.001 & $2.432(2.233-2.658)$ & 0.001 \\
\hline Race (White vs Black) & $2.169(1.990-2.365)$ & 0.001 & $1.770(1.611-1.944)$ & 0.001 \\
\hline \multicolumn{5}{|l|}{ Histology type } \\
\hline Papillary & I (Reference) & & I (Reference) & \\
\hline FV-PTC & $0.405(0.388-0.422)$ & 0.001 & $0.390(0.372-0.408)$ & 0.001 \\
\hline Follicular & $0.164(0.145-0.185)$ & 0.001 & $0.128(0.112-0.146)$ & 0.001 \\
\hline \multicolumn{5}{|l|}{ Extrathyroidal extension } \\
\hline None & I (Reference) & & I (Reference) & \\
\hline Capsule & 1.204 (I.127-1.287) & 0.001 & $1.238(1.151-1.330)$ & 0.001 \\
\hline Minimal & $5.314(5.083-5.556)$ & 0.001 & $3.881(3.696-4.074)$ & 0.001 \\
\hline Gross & 8.595 (7.839-9.423) & 0.001 & $5.797(5.240-6.412)$ & 0.001 \\
\hline Multifocality & $2.099(2.030-2.170)$ & 0.001 & $1.808(1.74 \mid-1.876)$ & 0.001 \\
\hline Distant metastasis ${ }^{\mathrm{a}}$ & $5.905(5.175-6.738)$ & 0.001 & $5.344(4.543-6.286)$ & 0.001 \\
\hline
\end{tabular}

Note: ancluding the lungs, bones, and brain.

Abbreviations: $\mathrm{Cl}$, confidence interval; DTC, differentiated thyroid cancer; FV-PTC, follicular variant of papillary thyroid cancer; LNM, lymph node metastasis; OR, odds ratio; SEER, Surveillance Epidemiology and End Results.

ETE, multifocality, and distant metastasis. Compared with microcarcinoma (1-10 mm), the risk of LNM was significantly higher for larger tumors. For primary tumors, the OR increased with each $10 \mathrm{~mm}$ increment, except for the largest tumor group ( $\geq 50 \mathrm{~mm}$ ) (Table 2 ).

After separating LNM into central lymph node metastasis (CLNM) and LLNM, multivariable logistic regression analysis indicated that size is a predictor for both types of metastasis. Also, as the tumor size increased by $10 \mathrm{~mm}$, patients were more likely to be diagnosed with LLNM than CLNM, which was consistent with the incremental ORs for LLNM calculated when adjusting for sex, age, race, histological type, ETE, multifocality, and distant metastasis in multivariable analyses (Table 3).

\section{Prognostic model for cervical LNM}

The final method for evaluating the relationships among tumor size, other predictors, and the probability of LNM consisted of constructing a model. A predictive model not only sheds light on the effect of each predictor on the probability of response according to the levels of other factors, but also allows for the quick estimation of the probability of response for individual subjects. For convenience, we defined the response to be $Y=0$ or 1 , with $Y=1$ corresponding to the positive development of LNM. The significant predictors identified by multivariable logistic regression were integrated into a statistical model that estimated the relationship between tumor size and LNM occurrence in DTC patients (Figure 1A). The values of the predictors derived from the coefficients of logistic regression and the regression equation for LNM risk were given by the following equations:

$$
\operatorname{Prob}\{\mathrm{LNM}=1\}=\frac{1}{1+\exp (-\mathrm{X} \beta)}, \quad \text { where }
$$

$$
\begin{aligned}
X \hat{\beta}= & -0.2256587+0.2165784 * \text { race }-0.5062728 * \text { sex } \\
& -0.9787501 * \text { histology }+1.579782 * \text { distant metastasis } \\
& +0.2068364 * \text { size }+0.268103 * \text { capsule }+1.444176 \\
& * \text { minimal ETE }+1.815767 * \text { gross ETE }+0.6347333 \\
& * \text { multifocality }-0.7181659 * \text { age }
\end{aligned}
$$

Distant metastasis, ETE, multifocality, above 45 year $=1$, 0 otherwise; size $(1-10 \mathrm{~mm})=1,(11-20 \mathrm{~mm})=2$, $(21-30 \mathrm{~mm})=3,(31-40 \mathrm{~mm})=4$, size $(41-50 \mathrm{~mm})=5$, $($ above $51 \mathrm{~mm})=6$; race $($ black $)=1,($ white $)=2,($ other $)=3$; histology $($ PTC $)=1,($ FV-PTC $)=2,($ FTC $)=3$ 
Table 3 Multivariate logistic analysis of tumor size in DTC according to the location of LNM

\begin{tabular}{|c|c|c|c|c|}
\hline \multirow{2}{*}{$\begin{array}{l}\text { Tumor } \\
\text { size }\end{array}$} & \multicolumn{2}{|l|}{ CLNM (NIa) } & \multicolumn{2}{|l|}{ LLNM (NIb) } \\
\hline & OR (95\% CI) & $P$-value & OR (95\% CI) & $P$-value \\
\hline $1-10$ & I (Reference) & & I (Reference) & \\
\hline $11-20$ & $2.182(2.058-2.313)$ & 0.001 & $1.618(1.512-1.730)$ & 0.001 \\
\hline $21-30$ & $2.514(2.350-2.689)$ & 0.001 & $1.926(1.784-2.079)$ & 0.001 \\
\hline $31-40$ & $2.525(2.3 \mid 7-2.752)$ & 0.001 & $2.005(1.82 I-2.206)$ & 0.001 \\
\hline $4 I-50$ & $2.260(2.027-2.520)$ & 0.001 & $2.433(2.175-2.722)$ & 0.001 \\
\hline$\geq 50$ & $1.688(1.5 \mid 4-1.883)$ & 0.001 & $2.616(2.359-2.902)$ & 0.001 \\
\hline
\end{tabular}

Note: $P$-value for OR was adjusted for sex, age, race, histology type, extension, multifocality, and distant metastasis as covariates.

Abbreviations: $\mathrm{Cl}$, confidence interval; CLNM, central lymph node metastasis; DTC, differentiated thyroid cancer; LNM, lymph node metastasis; LLNM, lateral lymph node metastasis; OR, odds ratio.
The outputs of the validation indicated that there was minor overfitting. However, the overfitting would have been worse if the risk factors had not been as robust. The closeness of the calibration curve to the $45^{\circ}$ line in Figure 1B demonstrates excellent validation on an absolute probability scale. However, the extent of missing data casts some doubt on the validity of this model and on the efficiency of its parameter estimates.

\section{Comparison of tumor size-based stratification for survival in DTC}

The mean follow-up time was $44.5 \pm 33.8$ months, with a range of $0-107$ months. The CSS rate was $98.6 \%$ at 5 years

A
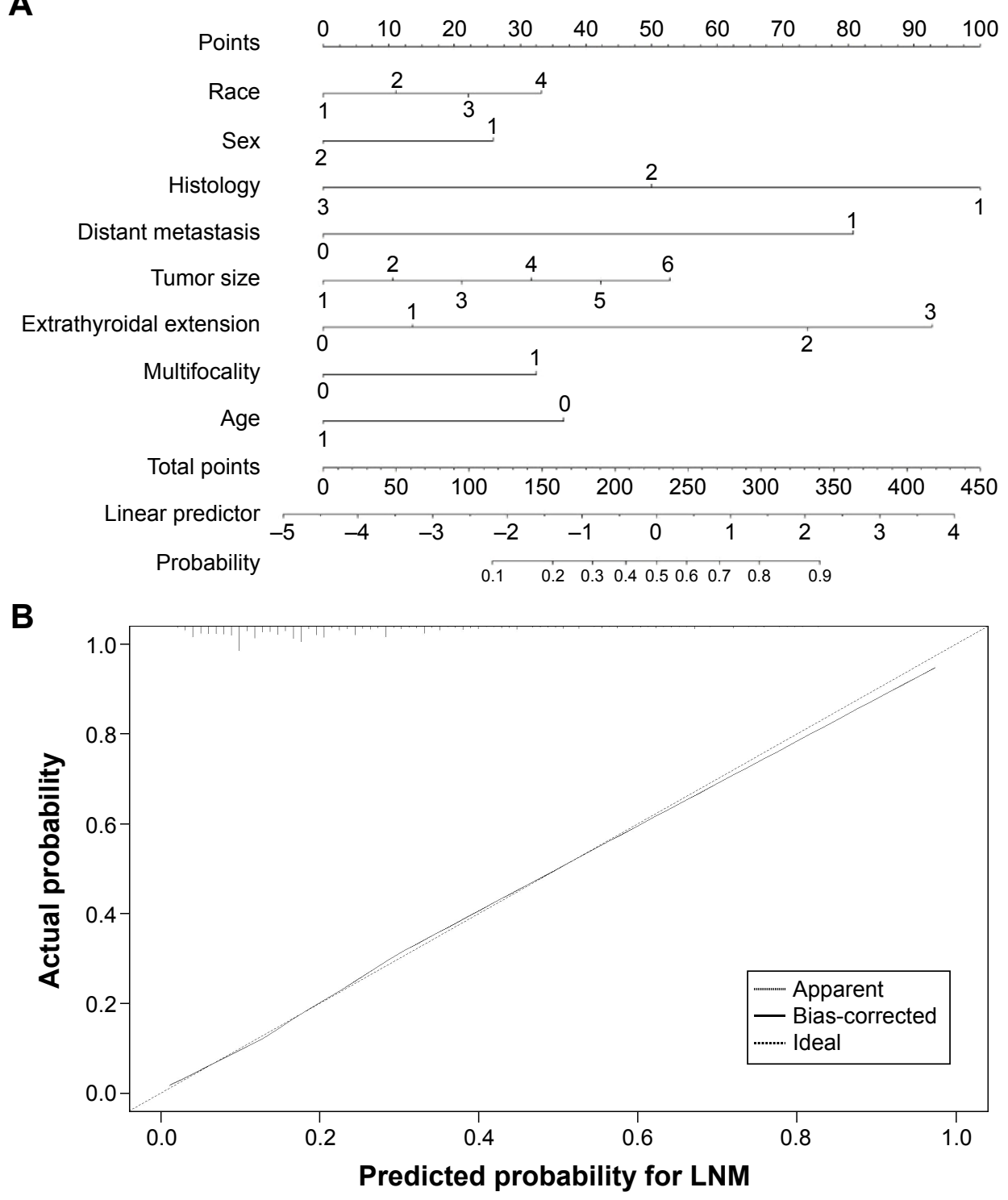

Figure I Lymph node metastasis predictive model.

Notes: (A) A model selection was performed by a backward stepdown selection process with the Akaike information criterion. (B) The discrimination and calibration of the system were assessed and internally validated, using bootstrapping techniques.

Abbreviation: LNM, lymph node metastasis. 

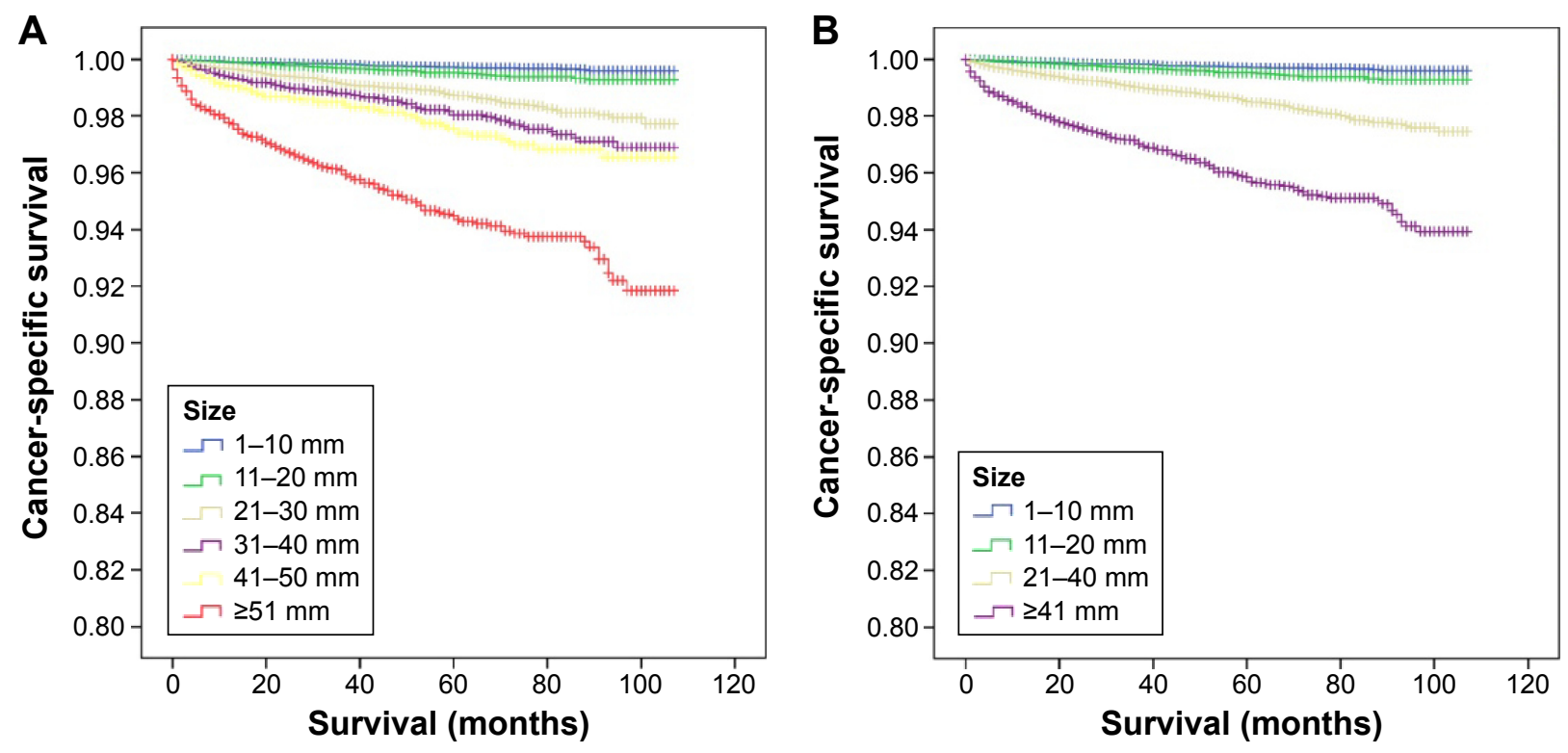

Figure 2 Log-rank tests of cancer-specific survival comparing among different tumor size groups. Notes: (A) $10 \mathrm{~mm}$ incremental classification. (B) 20 and $40 \mathrm{~mm}$ tumor sizes (10 mm for Tla and Tlb disease).

and $93.6 \%$ at 8 years from the time of the initial surgery. Although patients with DTC had a relatively favorable prognosis, we observed a continual decrease in CSS rates with increasing tumor size by Kaplan-Meier curves, with the largest ( $\geq 51 \mathrm{~mm}$ ) tumor group having the lowest survival rates. As shown in Figure 2A, the 8-year CSS rates by tumor size were as follows: $1-10 \mathrm{~mm}, 99.6 \% ; 11-20 \mathrm{~mm}, 99.3 \%$; 21-30 mm, 97.9\%; 31-40 mm, 96.9\%; 41-50 mm, 96.6\%; and $\geq 51 \mathrm{~mm}, 91.8 \%$. Compared with the current TNM classification, which uses the primary tumor size cutoffs of 20 and $40 \mathrm{~mm}$ (10 $\mathrm{mm}$ for T1a and T1b disease) to stratify subgroups for CSS (Figure 2B), the classification of $10 \mathrm{~mm}$ incremental intervals used in this study produced larger $\chi^{2}$ log-rank values $(1,045.965$ vs 867.914$)$, which is suggestive of a more precise prediction of CSS.

\section{Discussion}

LNM is considered a risk factor for poor clinical outcome and is common in patients with DTC. However, incidence rates vary widely, depending on the method of LNM detection. In the current literature, it is reported that prophylactic lymph node dissections (LNDs) yield high rates of lymph node micrometastases $(53 \%-65 \%) .{ }^{18-20}$ However, in the American Thyroid Association management guidelines for patients with DTC ${ }^{21}$ prophylactic central LND is recommended for patients with clinically uninvolved central neck lymph nodes (cN0) who have advanced primary tumors (T3 or T4), or clinically involved lateral neck nodes (cN1b) that may lead to macrometastases (ie, LNMs that are detected by preoperative ultrasound or during surgery). Such cases occurred only in approximately $22.5 \%$ of our cohort of patients with DTC. Because of the controversies regarding the application of prophylactic neck dissection and its relation to locoregional control and survival, predictability for LNM using a series of high-risk factors could facilitate the decision of whether to perform LND for more complete staging and reduction of "second-look" morbidity.

Tumor size is a critical prognostic feature of cervical $\mathrm{LNM},{ }^{19,22}$ and it is significantly associated with LNM in patients with DTC. According to our data, adverse clinical features (including male sex and white race) and disease aggressiveness (including the occurrence of gross ETE and distant metastasis) became more prevalent as the tumor size increased, which greatly explains the association between tumor size and LNM. Multivariate analysis further demonstrated that male sex, older age ( $\geq 45$ years), non-Hispanic white race, papillary histology type, ETE, multifocality, and distant metastasis are the important factors associated with larger tumor size in predicting histologically confirmed LNM.

Interestingly, our data showed that the presence of LNM in follicular TC or follicular variant of papillary TC at diagnosis was unlikely to be papillary TC. These two histological types have been consistently associated with increased mortality and poorer outcome. Their wide invasiveness is probably attributable to the distinct biological features of Hurthle cell carcinomas from papillary TC, such as vascular or capsular invasion and hematogenous spread. 
The multistratification of tumor size developed in this study to evaluate the effect of size on LNM at initial treatment is a novel addition to the current understanding of the $\mathrm{T}$ stage. When we set tumor size intervals of $10 \mathrm{~mm}$ as reference, we found that the larger the tumor, the higher the incidence of LNM. The risk trend of LNM reached significance with the $10 \mathrm{~mm}$ interval size stratification, up to $50 \mathrm{~mm}$. When the tumor size is $>50 \mathrm{~mm}$, the composition of Hurthle cells in carcinomas becomes higher, which may promote distant metastasis. In particular, a large tumor size is a strong indicator of LLNM, given the sequential pattern of LNM in DTC spreading from the thyroid gland and the central and lateral lymph node compartments. The extended cervical metastasis of LLNM reflects the greater tendency of larger tumors to metastasize distantly from a primary location than do smaller tumors.

Based on our finding of the association between stratified tumor size and LNM, we designed a model that systematically considers multiple variables to estimate an individual risk of LNM in patients with DTC. Our nomogram employed easily accessible clinical information with high concordance on an absolute probability scale. Coefficients from the model indicated a change of $\log (\mathrm{OR})$ per unit increment in tumor size (relative to a reference range of $1-10 \mathrm{~mm}$ ) when the tumor size represented a single factor that is linear and does not interact with other factors, if all other factors remained constant. According to the model designed in this study, for six cases with primary tumors ranging in size from 5 to $55 \mathrm{~mm}$ with $10 \mathrm{~mm}$ increments, the extra risk of LNM deriving from tumor size would increase from $0.2068364 \times 1$ to $0.2068364 \times 6$, if other variables remained constant.

Tumor size is also closely associated with disease outcome, including both tumor recurrence and cancerspecific mortality rates. ${ }^{23,24}$ In the present study, we found that the 8-year cumulative survival rates for patients with DTC decreased gradually and significantly from $99.6 \%$ for $1-10 \mathrm{~mm}$ tumors to $91.8 \%$ for tumors $\geq 51 \mathrm{~mm}$. The $10 \mathrm{~mm}$ incremental classification produced larger $\chi^{2}$ values than the traditional system for primary tumors, which may facilitate decisions about the extent of LND at the initial surgery. Also, this information may assist clinicians and patients to objectively and confidently estimate the risk of disease outcomes based on tumor size.

Although the management of clinically negative DTC is controversial, tumor size and this predictive model could provide more information on LNM associated with other imaging methods. The present findings suggest that in cases where these predictive factors are available, the incidence of neck metastases may be high and further evaluation regarding the risks and benefits of LND may be warranted, at least for the lateral compartment.

\section{Limitations}

Our study has some limitations. First, the confirmation for LNM was mainly based on pathological analysis of samples collected during therapeutic LND. The low incidence of LNM in this cohort suggests the possible underestimation of LNM in patients with DTC. However, previous studies have shown that ultrasonography is an important tool in the detection of metastatic nodes, with high specificity and positive predictive value. Therefore, the current ultrasonography system is relatively accurate for the diagnosis of clinical LNM and seems to be improved by our findings and the model presented herein, which integrates a series of clinicopathological variables. Second, in patients with LLNM from the SEER database, we could not differentiate the status of the central lymph node. Although CLNM is reported to predict LLNM, ${ }^{20,25}$ our risk evaluation for LLNM did not identify CLNM as a risk factor on multivariate analysis. Diagnostic algorithms for LNM suggested by this analysis have not been tested and will require further testing in a prospective study before recommendations can be made. Finally, given the dramatic shift in the relative incidence of these lesions over time, continued reevaluation must be applied to the risk calculator to ensure accuracy in future populations.

\section{Conclusion}

Larger tumors are more likely to have aggressive features and locoregional metastases than smaller ones. With multistratifications for tumor size based on $10 \mathrm{~mm}$ increments, LNM risk was found to increase significantly relative to the reference range $(1-10 \mathrm{~mm})$, particularly for LLNM. The predictive model indicates an extra change of $\log (\mathrm{OR})$ for $\mathrm{LNM}$ as 0.2 per unit increment in tumor size based on the multivariable logistic regression analysis. These findings indicate that the model proposed herein can allow clinicians caring for patients with DTC to objectively estimate the likelihood of LNM before surgery and optimize the use of therapeutic modalities for evaluating cervical lymph nodes.

\section{Acknowledgments}

The authors acknowledge the efforts of the Surveillance, Epidemiology, and End Results (SEER) Program tumor registries in the creation of SEER database. The interpretation and reporting of these data are the sole responsibility of the authors. This work was supported by 
funds from the National Natural Science Foundation of China (Nos 81572622 and 81272934 to QHJ) and Science and Technology Commission of Shanghai Municipality (No 14ZR1407300 to YW).

\section{Disclosure}

The authors report no conflicts of interest in this work.

\section{References}

1. McLeod DS, Sawka AM, Cooper DS. Controversies in primary treatment of low-risk papillary thyroid cancer. Lancet. 2013;381(9871): 1046-1057.

2. Siegel RL, Miller KD, Jemal A. Cancer statistics, 2015. CA Cancer J Clin. 2015;65(1):5-29.

3. Konturek A, Barczynski M, Stopa M, Nowak W. Trends in prevalence of thyroid cancer over three decades: a retrospective cohort study of 17,526 surgical patients. World J Surg. 2016;40(3):538-544.

4. Gilliland FD, Hunt WC, Morris DM, Key CR. Prognostic factors for thyroid carcinoma. A population-based study of 15,698 cases from the Surveillance, Epidemiology and End Results (SEER) program 1973-1991. Cancer. 1997;79(3):564-573.

5. Lim YS, Lee JC, Lee YS, et al. Lateral cervical lymph node metastases from papillary thyroid carcinoma: predictive factors of nodal metastasis. Surgery. 2011;150(1):116-121.

6. Schneider DF, Chen H, Sippel RS. Impact of lymph node ratio on survival in papillary thyroid cancer. Ann Surg Oncol. 2013;20(6):1906-1911.

7. Lang BH, Ng SH, Lau LL, Cowling BJ, Wong KP, Wan KY. A systematic review and meta-analysis of prophylactic central neck dissection on short-term locoregional recurrence in papillary thyroid carcinoma after total thyroidectomy. Thyroid. 2013;23(9):1087-1098.

8. Kim KM, Park JB, Bae KS, Kang SJ. Analysis of prognostic factors in patients with multiple recurrences of papillary thyroid carcinoma. Surg Oncol. 2012;21(3):185-190.

9. Sakorafas GH, Sampanis D, Safioleas M. Cervical lymph node dissection in papillary thyroid cancer: current trends, persisting controversies, and unclarified uncertainties. Surg Oncol. 2010;19(2):e57-e70.

10. Cohen R, Campos JM, Salaun C, et al. Preoperative calcitonin levels are predictive of tumor size and postoperative calcitonin normalization in medullary thyroid carcinoma. Groupe d'Etudes des Tumeurs a Calcitonine (GETC). J Clin Endocrinol Metab. 2000;85(2):919-922.

11. Machens A, Holzhausen HJ, Dralle H. The prognostic value of primary tumor size in papillary and follicular thyroid carcinoma. Cancer. 2005;103(11):2269-2273.
12. Sobin LH GM, Wittekind CH. UICC: TNM Classification of Malignant Tumors. 7th ed. New York: Wiley-Liss; 2009.

13. Edge SB, Compton CC. The American Joint Committee on Cancer: the 7th edition of the AJCC cancer staging manual and the future of TNM. Ann Surg Oncol. 2010;17(6):1471-1474.

14. Choi N, Moon WJ, Lee JH, Baek JH, Kim DW, Park SW. Ultrasonographic findings of medullary thyroid cancer: differences according to tumor size and correlation with fine needle aspiration results. Acta Radiol. 2011;52(3):312-316.

15. Warren JL, Klabunde CN, Schrag D, Bach PB, Riley GF. Overview of the SEER-Medicare data: content, research applications, and generalizability to the United States elderly population. Med Care. 2002; 40(8 Suppl):IV-3-18.

16. Harrell FE, Lee KL, Mark DB. Multivariable prognostic models: issues in developing models, evaluating assumptions and adequacy, and measuring and reducing errors. Stat Med. 1996;15(4):361-387.

17. Nunez E, Steyerberg EW, Nunez J. [Regression modeling strategies]. Rev Esp Cardiol. 2011;64(6):501-507.

18. Park JY, Koo BS. Individualized optimal surgical extent of the lateral neck in papillary thyroid cancer with lateral cervical metastasis. Eur Arch Otorhinolaryngol. 2014;271(6):1355-1360.

19. Sanchez Fuentes MN, Miguelena Bobadilla JM, Casamayor Franco MC, Barranco Dominguez JI, Dobon Rascon MA. Lateral neck tumour: Ectopic thyroid vs metastasis of a differentiated thyroid carcinoma. Cir Esp. 2015;93(9):e115-e117.

20. Ducoudray R, Tresallet C, Godiris-Petit G, Tissier F, Leenhardt L, Menegaux F. Prophylactic lymph node dissection in papillary thyroid carcinoma: is there a place for lateral neck dissection? World J Surg. 2013;37(7):1584-1591.

21. Haugen BR, Alexander EK, Bible KC, et al. 2015 American Thyroid Association Management Guidelines for adult patients with thyroid nodules and differentiated thyroid cancer. Thyroid. 2016;26(1):1-133.

22. Kim YS. Patterns and predictive factors of lateral lymph node metastasis in papillary thyroid microcarcinoma. Otolaryngol Head Neck Surg. 2012;147(1):15-19.

23. Roh JL, Park JY, Rha KS, Park CI. Is central neck dissection necessary for the treatment of lateral cervical nodal recurrence of papillary thyroid carcinoma? Head Neck. 2007;29(10):901-906.

24. Hovens GC, Stokkel MP, Kievit J, et al. Associations of serum thyrotropin concentrations with recurrence and death in differentiated thyroid cancer. J Clin Endocrinol Metab. 2007;92(7):2610-2615.

25. Kim HJ, Sohn SY, Jang HW, Kim SW, Chung JH. Multifocality, but not bilaterality, is a predictor of disease recurrence/persistence of papillary thyroid carcinoma. World J Surg. 2013;37(2):376-384.
OncoTargets and Therapy

\section{Publish your work in this journal}

OncoTargets and Therapy is an international, peer-reviewed, open access journal focusing on the pathological basis of all cancers, potential targets for therapy and treatment protocols employed to improve the management of cancer patients. The journal also focuses on the impact of management programs and new therapeutic agents and protocols on
Dovepress

patient perspectives such as quality of life, adherence and satisfaction. The manuscript management system is completely online and includes a very quick and fair peer-review system, which is all easy to use. Visit http://www.dovepress.com/testimonials.php to read real quotes from published authors. 